\title{
Potencial fungitóxico do óleo essencial de Piper hispidinervum (pimenta longa) sobre os fungos fitopatogênicos Bipolaris sorokiniana, Fusarium oxysporum e Colletotrichum gloeosporioides
}

\author{
Lidiany Mendonça ZACARONI ${ }^{1}$, Maria Graças CARDOSO ${ }^{2}$, Paulo Estevão SOUZAª , Flavio Araujo \\ PIMENTEL ${ }^{4}$, Luiz Gustavo de Lima GUIMARÃES 5 , Ana Paula Soares Pinto SALGADO5
}

\begin{abstract}
RESUMO
O objetivo deste trabalho foi avaliar a atividade fungicida in vitro do óleo essencial das folhas de Piper hispidinervum sobre Bipolaris sorokiniana, Fusarium oxysporum e Colletotrichum gloeosporioides. Para os ensaios biológicos, empregou-se o teste bioanalítico in vitro utilizando as concentrações de 100, 200, 500, 1000, 1500 e $2000 \mu \mathrm{g} \cdot \mathrm{mL}^{-1}$ do óleo essencial. Estas foram incorporadas no meio de cultura BDA (batata-dextrose-ágar) para avaliação do crescimento ou inibição micelial. O delineamento estatístico utilizado foi o inteiramente casualizado, com quatro repetições. $\mathrm{Na}$ concentração de $200 \mu \mathrm{g}$. $\mathrm{mL}^{-1}$, observou-se uma inibição total do fitopatógeno Bipolaris sorokiniana enquanto que, para o Fusarium oxysporum e o Colletotrichum gloeosporioides esta ocorreu na concentração de $1000 \mu \mathrm{g} \cdot \mathrm{mL}^{-1}$.
\end{abstract}

PalaVRaS-CHAVE: Piper hispidinervum, Fitopatógenos, Doenças de plantas.

\section{Fungitoxic potential of the essential oil the Piper hispidinervum (long- pepper) against phytopathogenic fungi Bipolaris sorokiniana, Fusarium oxysporum e Colletotrichum gloeosporioides}

\begin{abstract}
The objective of this study was to evaluate the in vitro antifungal activity of the essential oil of the leaves of Piper hispidinervum against Bipolaris sorokiniana, Fusarium oxysporum and Colletotrichum gloeosporioides. For the biological tests, using the bioanalitic test in vitro the concentrations of 100,200, 500, 1000, 1500 and $2000 \mu \mathrm{g} \cdot \mathrm{mL}^{-1}$ the essential oil. This were incorporated into PDA (potato dextrose agar) medium in order to evaluate fungal mycelial growth or inhibition. The statistic design used was completely randomized, with four replicates. In the concentration of $200 \mu \mathrm{g} . \mathrm{mL}^{-1}$, observed inhibited complete the phytopathogens Bipolaris sorokiniana while the Fusarium oxysporum and Colletotrichum gloeosporioides this is occurred in the concentration of $1000 \mu \mathrm{g} \cdot \mathrm{mL}^{-1}$.
\end{abstract}

KEY WORDS: Piper hispidinervum, phytopathogens, plant disease.

\footnotetext{
1 Universidade Federal de Lavras, Departamento de Química, Universidade Federal de Lavras, Campus Universitário, Caixa Postal 3037, CEP 37200-000, Lavras, Minas Gerais. e-mail: lidyz@bol.com.br

2 Universidade Federal de Lavras, Departamento de Química, Campus Universitário, Caixa Postal 3037, CEP 37200-000, Lavras, Minas Gerais. e-mail: mcardoso@ufla.br

3 Universidade Federal de Lavras, Departamento de Fitopatologia, Campus Universitário, Caixa Postal 3037, CEP 37200-000, Lavras, Minas Gerais. e-mail: pauleste@ufla.br

${ }^{4}$ Embrapa CNPAT, CP 3761, 60511-110 Fortaleza - CE, Brasil. e-mail: fflavio.pimentel@cnpat.embrapa.br

${ }^{5}$ Departamento de Química, Universidade Federal de Lavras, Campus Universitário, Caixa Postal 3037, 37200-000, Lavras, Minas Gerais. e-mail: luizufop@yahoo.com.br
} 


\section{INTRODUÇÃO}

A utilização de produtos naturais no controle de doenças de plantas vem se tornando uma alternativa promissora para reduzir o uso indiscriminado de defensivos agrícolas. Segundo Pozza et al. (1999), os fungos são responsáveis por $70 \%$ das doenças que causam danos em várias culturas, reduzindo a produtividade.

Diversas espécies de Fusarium, comumente são fitopatógenos e saprófitos do solo. Alguns grupos habitam as regiōes aéreas superiores de plantas. A maior parte das espécies do gênero parasitam sementes de cereais e outros frutos do campo, causando danos antes e após a colheita. O fungo Bipolaris sorokiniana, é um fitopatógeno causador de manchas em folhas de seringueira, coqueiro, bananeira, arroz, cana-de-açúcar e é o responsável pela queima de folhas de milho e sorgo. Uma das principais doenças causadas por este patógeno é a mancha marron causada em culturas de trigo. O Colletotrichum gloeosporioides é responsável pela antracnose pós-colheita em frutos e pela antracnose foliar em diversas hortaliças, entre outras. (Kimati et al, 2005).

Diversas plantas têm revelado atividade fungicida com várias substâncias já isoladas. Dentre estas, cita-se os trabalhos de Salgado et al. (2003), que demonstraram o efeito fungicida de óleos essenciais de três espécies de eucalipto (E. urophylla, E. citriodora e E. camaldulensis) sobre os fitopatógenos (Fusarium oxysporum, Botrytis cinerea e Bipolaris sorokiniana). Em seus estudos observaram atividades fungitóxicas para a concentração de $500 \mathrm{mg} / \mathrm{L}$ das três espécies de eucalipto, porém o óleo essencial da espécie Eucalyptus urophylla apresentou maior efeito inibitório no crescimento micelial das três espécies fúngicas.

Mendonça (2004), estudando o efeito dos óleos essenciais de cravo-da-índia, orégano e manjericão sobre Staphylococcus aureus, verificou efeito inibitório a partir das concentraçōes de 1,5 e $10 \%$, respectivamente. Pereira (2008), analisando os óleos essenciais de cravo-da-índia e orégano, observou atividade fungicida sobre o fungo Penicillium commune, com inibição total no crescimento a partir das concentrações de $250 \mathrm{mg} . \mathrm{L}^{-1}$ e $2000 \mathrm{mg} . \mathrm{L}^{-1}$, respectivamente.

Piper hispidinervum, conhecida popularmente por pimenta longa, é um arbusto da família Piperaceae, encontrada em condiçōes silvestres no vale do rio Acre. Trata-se de uma espécie que apresenta regeneração abundante e alta capacidade de rebrota após o corte. Dados de Miranda (2002) indicam que seu óleo essencial é rico em safrol (aproximadamente 92\%). Esta substância concentra-se principalmente nas folhas e nos ramos secundários, podendo ser extraído por processo de hidrodestilação.

Segundo Sá e colaboradores (2004) a pimenta longa se destaca por sua importância econômica, devido à utilização do safrol como matéria-prima pelas indústrias químicas para a síntese de heliotropina (piperonal, usado como fixador de fragâncias) e butóxido de piperonila (usado como agente sinergístico junto com o piretrum).

$\mathrm{Na}$ busca por produtos naturais que atuem como fungicidas controlando doenças de plantas que afetam diversas culturas, o presente trabalho teve por objetivo avaliar o efeito fungitóxico do óleo essencial de Piper hispidinervum sobre os fitopatógenos Fusarium oxysporum cubensis, Colletotrichum gloeosporioides e Bipolaris sorokiniana.

\section{MATERIAIS E MÉTODOS}

As folhas de pimenta longa foram coletadas em maio de 2005 de plantas cultivadas no Horto de Plantas Medicinais da Universidade Federal de Lavras (UFLA), situada na cidade de Lavras - MG, localizada a uma latitude $21^{\circ} 14^{\prime} 43$ sul e a uma longitude $44^{\circ} 59^{\prime \prime} 59$ oeste, estando a uma altitude de 919 metros.

A extração do óleo essencial das folhas de pimenta longa foi realizada por meio da hidrodestilação, utilizando-se aparelho de Clevenger modificado (Castro 2006). Coletou-se o hidrolato, que em seguida foi centrifugado em centrífuga cruzeta horizontal a 965,36 x G por 5 minutos. O óleo essencial foi retirado com o auxílio de uma micropipeta e acondicionado em frasco de vidro âmbar envolto com papel alumínio, mantendo-o sobre refrigeração.

As análises químicas do óleo essencial de P. hispidinervum foram realizadas no Laboratório de Química Orgânica, no Departamento de Química da UFLA, empregando a técnica de cromatografia em fase gasosa (CG). Utilizou-se um equipamento Shimadzu CG - 17A, nas seguintes condiçōes experimentais: coluna capilar (DB5) constituída de (5\%-fenil, $95 \%$ dimetilpolisiloxano); temperatura do injetor $200{ }^{\circ} \mathrm{C}$; temperatura do detector de ionização de chama (DIC) 210 ${ }^{\circ} \mathrm{C}$; programação da coluna com temperatura de $130^{\circ} \mathrm{C} / 4 \mathrm{~min}$. elevando-se de $130^{\circ} \mathrm{C}$ para $150^{\circ} \mathrm{C}$, aumentando $5^{\circ} \mathrm{C} / \mathrm{min}$, até atingir $150^{\circ} \mathrm{C}$. Foi utilizado como gás de arraste o nitrogênio $(0,95 \mathrm{~mL} / \mathrm{min})$ e a razão de separação de $1: 100$. O volume injetado de amostra foi $1 \mu \mathrm{L}$ diluído na razão de 1:40 (v/v) em diclorometano. A análise qualitativa foi realizada por meio de padronização externa, utilizando padrão de safrol (Acros, 97\%), diluído na razão de 1:50 (v/v) em diclorometano. A quantificação foi obtida por integração eletrônica, pela técnica de normalização de áreas.

As culturas utilizadas para os testes do potencial fuingitóxico do óleo essencial foram obtidas na Micoteca do Departamento de Fitopatologia da UFLA e os números de registro para cada espécie são: CML-1450 (B. sorokiniana), CML-1451 (F. oxysporum) e CML-1473 (C. gloeosporioides). 
Os ensaios biológicos foram realizados no Laboratório de Micologia do Departamento de Fitopatologia. A partir do óleo essencial obtido, foram preparadas concentraçōes 100 , 200, 500, 1000, 1500 e $2000 \mu \mathrm{g} \cdot \mathrm{mL}^{-1}$ em éter etílico, seguido da incorporação destas ao meio de cultura BDA (batata, dextrose, ágar) previamente autoclavado e semi-fundente. Foram preparadas placas testemunhas com o meio BDA sem adição de solvente (testemunha absoluta) e com éter etílico (testemunha relativa) para determinar o efeito deste nos tratamentos com o óleo essencial de P. hispidinervum.

Em uma capela de fluxo laminar, concentrações do óleo, previamente solubilizadas em éter etílico foram adicionadas ao meio de cultura (BDA), fundente, empregando o método de diluição em ágar e vertidas em placas de Petri de $9 \mathrm{~cm}$ previamente esterilizadas. Um disco invertido de $8 \mathrm{~mm}$ de diâmetro, contendo o micélio de cada fungo (retirado da colônia com doze dias em BDA) foi depositado no centro de cada placa. Realizou-se o mesmo procedimento com as testemunhas absoluta e relativa. As placas foram seladas com papel aderente, identificadas e incubadas em câmara de germinação sob fotoperíodo de $12 \mathrm{~h}$ à temperatura de $25^{\circ} \mathrm{C}$ por dez dias (Salgado et al., 2003).

A avaliação do experimento iniciou 24 h após sua instalação, realizando-se mediçōes ortogonais do diâmetro das colônias diariamente, sendo que cada medição correspondeu à média de duas medidas diametralmente opostas da colônia fúngica, tendo como referência as placas testemunhas (absoluta e relativa). O índice de crescimento micelial (ICM) ou taxa de crescimento micelial (TCM) foi calculado pela fórmula modificada de Nakagava Maguire, adaptada por Salgado et al. (2003).

$$
\mathrm{ICM}=\frac{C 1}{N 1}+\frac{C 2}{N 2}+\frac{C n}{N n}
$$

Onde:

$C 1, C 2, C n=$ crescimento micelial das colônias na primeira, segunda e última avaliação.

$$
N 1, N 2, N n=\text { número de dias. }
$$

Adotou-se o esquema fatorial com quatro repetições, em delineamento experimental inteiramente casualizado (DIC). Os resultados foram analisados estatisticamente pelo método ANAVA, comparando-se as médias do crescimento micelial pelo teste Scott-Knott ao nível de $5 \%$ de probabilidade.

\section{RESULTADOS E DISCUSSÃO}

A análise química do óleo essencial de $P$. hispidinervum, empregando-se a cromatografia em fase gasosa, apresentou como composto majoritário o safrol (89\%) (Figura 1). Resultado similar foi observado por Gottilieb et al. (1981), onde o teor de safrol encontrado foi de $89 \%$.

Bergo \& Silva (1999), trabalhando com a pimenta longa constataram a presença de safrol como composto majoritário em seu óleo essencial, alcançando um teor de 90 a $94 \%$. Miranda (2002) observou um teor de safrol superior a $92 \%$ no óleo essencial de pimenta longa, coletadas na região amazônica. Pesquisas de Fazolin et al. (2007), mostraram que o óleo essencial extraído de plantas dessa região apresenta um teor de safrol superior a 90\%. Essas variaçōes no teor de safrol encontrado na planta podem estar diretamente relacionadas ao horário e local de coleta, bem como as condiçôes do solo e do clima em que foi cultivada (Simões et al., 2004).

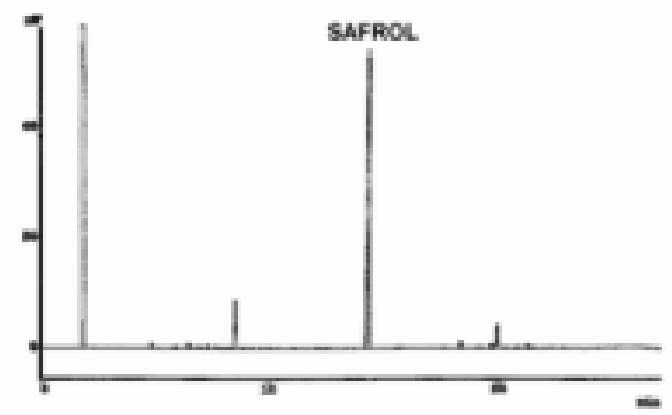

Figura 1 - Cromatograma (CG) do óleo essencial extraído de folhas frescas de Piper hispidinervum.

O efeito das concentrações do óleo essencial das folhas de $P$. hispidinervum sobre o crescimento micelial dos fitopatógenos B. sorokiniana, F. oxysporum e C. gloeosporioides pode ser observado pelos dados descritos na Tabela 1. Observa-se que para todos os fungos, houve inibição do crescimento micelial com doses crescentes do óleo essencial, portanto, os resultados diferiram na concentração que inibiu totalmente o crescimento micelial. Para a espécie B. sorokiniana a inibição total ocorreu a uma concentração de $200 \mu \mathrm{g} \cdot \mathrm{mL}^{-1}$, enquanto que para as espécies $F$. oxysporum e $C$. gloeosporioides foi observado o mesmo efeito, porém, na concentração de 1000 $\mu \mathrm{g} \cdot \mathrm{mL}^{-1}$.

Parmar et al. (1997) isolaram e caracterizaram quimicamente os arilpropanóides em extratos brutos e em óleos essenciais de plantas da família Piperaceae encontrando substâncias como safrol, miristicina, eugenol, dillapiol e apiol. Esses compostos apresentam propriedades antimicrobianas e antioxidantes assim como, efeitos citotóxicos e psicotrópicos. Portanto, pode-se inferir relações entre o efeito fungicida observado no óleo essencial de Piper hispidinervum e a presença de safrol como seu componente majoritário. 
Tabela 1 - Médias do crescimento micelial dos fungos Bipolaris sorokiniana, Fusarium oxysporium, Colletotrichum gloeosporióides nos diferentes tratamentos.

\begin{tabular}{lccc}
\hline \multirow{2}{*}{ TRATAMENTO } & \multicolumn{1}{c}{ ICM $^{*}$} & C. gloeosporioides \\
\cline { 2 - 4 } & B. sorokiniana & F. oxysporium & $47,07 \mathrm{a}$ \\
\hline Testemunha absoluta & $58,96 \mathrm{a}$ & $50,52 \mathrm{a}$ & $48,84 \mathrm{a}$ \\
Testemunha relativa & $52,51 \mathrm{a}$ & $51,71 \mathrm{a}$ & $43,26 \mathrm{a}$ \\
$100 \mu \mathrm{g} / \mathrm{mL}$ & $23,57 \mathrm{~b}$ & $43,99 \mathrm{~b}$ & $11,13 \mathrm{~b}$ \\
$200 \mu \mathrm{g} / \mathrm{mL}$ & $0,00 \mathrm{c}$ & $15,09 \mathrm{c}$ & $7,66 \mathrm{~b}$ \\
$500 \mu \mathrm{g} / \mathrm{mL}$ & $0,00 \mathrm{c}$ & $14,60 \mathrm{c}$ & $0,00 \mathrm{c}$ \\
$1000 \mu \mathrm{g} / \mathrm{mL}$ & $0,00 \mathrm{c}$ & $0,00 \mathrm{~d}$ & $0,00 \mathrm{c}$ \\
$1500 \mu \mathrm{g} / \mathrm{mL}$ & $0,00 \mathrm{c}$ & $0,00 \mathrm{~d}$ & $0,00 \mathrm{c}$ \\
$2000 \mu \mathrm{g} / \mathrm{mL}$ & $0,00 \mathrm{c}$ & $0,00 \mathrm{~d}$ & \\
\hline
\end{tabular}

As médias seguidas de mesma letra não diferem significativamente entre si, pelo teste Scott-Knott ao nível de $5 \%$ de probabilidade. *ICM: Índice de crescimento micelial.

Bastos \& Albuquerque (2004), avaliaram o efeito do óleo essencial de $P$. aduncum no controle de C. musae, constatando inibição de $100 \%$ do crescimento micelial e da germinação de conídios, utilizando uma concentração de $100 \mu \mathrm{g} \cdot \mathrm{mL}^{-1}$. No teste in vivo apenas $1 \%$ da concentração do óleo essencial foi capaz de inibir a manifestação de podridōes nos frutos de banana. O mesmo efeito de inibição de crescimento micelial foi observado com o óleo essencial de P. hispidinervum sobre $B$. sorokiniana, porém, a uma concentração de 200 $\mu \mathrm{g} \cdot \mathrm{mL}^{-1}$. Os óleos essenciais dessas plantas apresentam em comum compostos da classe dos arilpropanóides, diferindo apenas no teor encontrado em cada planta. Hanada et al. (2004), estudando o efeito biológico do óleo essencial de $P$. hispidinervum observaram uma inibição parcial da germinação de conídios de Mycosphaerella fijiensis Morelet, atribuindo a esta a presença do safrol no seu óleo essencial.

Navickiene et al. (2006), relataram a alta atividade fungicida do óleo essencial de frutos das plantas $P$. aduncum e $P$. tuberculatum sobre os fitopatógenos Cladosporium cladosporioides e C. sphaerospermum, respectivamente, com CMI (concentração mínima inibitória) de $10 \mu \mathrm{g} \cdot \mathrm{mL}^{-1}$. Essas plantas também apresentam safrol em sua constituição, comprovando suas atividades antimicrobianas.

\section{CONCLUSÕES}

O óleo essencial de pimenta longa na concentração de 200 $\mu \mathrm{g} \cdot \mathrm{mL}^{-1}$, inibiu totalmente o crescimento do fitopatógeno Bipolaris sorokiniana enquanto que, para o Fusarium oxysporum e o Colletotrichum gloeosporioides esta ocorreu na concentração de $1000 \mu \mathrm{g} \cdot \mathrm{mL}^{-1}$.

\section{AGRADECIMENTOS}

Ao CNPq e FAPEMIG pelo apoio financeiro e bolsas concedidas.

\section{BIBLIOGRAFIA CITADA}

Bastos, C.N.; Albuquerque, P.S.B. 2004. Efeito do óleo de Piper aduncum no controle em pós-colheita de Colletotrichum musae em banana. Fitopatologia Brasileira, 29(5): 555-557.

Bergo, C.L.; Silva, M.R. 1999. Avaliação do efeito da época de corte da pimenta longa (Piper hispidinervum) no rendimento de óleo essencial. Boletim de pesquisa, 151. Embrapa- CPAF/ AC, Rio Branco, Acre.

Castro, D.P., Cardoso, M.G., Moraes, J.C., Santos, N.M., Baliza, D.P. 2006. Não preferência de Spodoptera frugiperda (Lepidóptera: Noctuidae) por óleos essenciais de Achillea millefolium L. e Thymus vulgarius L. Revista Brasileira de Plantas Medicinais, 8(4): $27-32$.

Fazolin, M.; Estrela, J.L.V.; Fazolin, M.; Catani, V.; Alécio, M.R.; Lima, M.S. 2007. Propriedade inseticida dos óleos essenciais de Piper hispidinervum C.DC.; Piper aduncum L. e Tanaecium nocturnum (Barb. Rodr.) Bur. \& k.shum sobre Tenebrio molitor L. 1758. Ciência e Agrotecnologia, 31(1): 113 - 120.

Gottlieb, O.R., Koketsu, M., Magalhães, M.T., Maia, J.G.S., Mendes, A.I. da Rocha, Da Silva, M.L., Wilberg, V.C. 1981. Óleos essenciais da Amazônia. VII. Acta Amazonica, 11:143148.

Hanada, R.E.; Gasparotto, L.; Pereira, J.C.R. 2004. Eficiência de desinfestantes na erradiação de conídios de Mycosphaerella fijiensis aderidos à superfície de bananas. Fitopatologia Brasileira, 29(1): $94-96$.

Kimati, H.; Amorim, A.B.F.; Camargo, L.E.A.; Rezende, J.A.M. 2005. Manual de Fitopatologia. Agronômica Ceres, São Paulo, SP, Brasil. 663pp.

Mendonça, A.T. 2004. Efeito dos óleos essenciais de condimentos sobre o crescimento de Staphylococcus aureus em ricota cremosa. Tese de Doutorado, Universidade Federal de Lavras, Lavras, Minas Gerais. 72pp.

Miranda, E.M. 2002. Caracterização e avaliação produtiva de uma população nativa de Pimenta Longa (Piper hispidinervum) no Seringal Cachoeira, AC. Brasil. Acta Amazonica, 32(1): 9-20. 
Navickiene, H.M.D.; Morandim, A.A.; Marques, M.O.M.; Yong, M.C.M.; Kato, M.J. 2006. Composition and antifungal activity of essential oils from Piper aduncum, Piper arboreum and Piper tuberculatum. Quimica Nova, 29(3): 467-470.

Parmar, V.S.; Jain, S.C.; Bisht, K.S; Jain, R.; Taneja, P.; Jha, A.; Tyagi, O.D.; Prasad, A.K.; Wengel J.; Olsen, C.E.; Boll, P.M. 1997. Phytochemistry of the genus Piper. Phytochemistry, 46(4): 597-673.

Pereira, A.A.; Cardoso, M.G.; Abreu, L.R.; Morais, A.R.; Guimarães, L.G.L.; Salgado, A.P.S.P. 2008. Caracterização química e efeito inibitório de óleos essenciais sobre o crescimento de Staphylococcus aureus e Escherichia coli. Ciência e Agrotecnologia, 32(3): 887-893.

Pozza, E.A.; Souza, P.E.; Castro, H.A.; Pozza, A.A.A. 1999. Freqüência da ocorrência de doenças da parte aérea de plantas na região de Lavras-MG. Ciência Agrotecnologia 23 (4): 1001-1005.
Sá, C.P.; Boyma, M.M.A.; Gomes, F.C.R.; Oliveira, E.L. 2004. Aspectos Agronômicos e Socioeconômicos do Cultivo da Pimenta longa para Produção de Safrol no Acre. Comunicado técnico, 164. Empraba-CPAF/AC, Rio Branco, Acre.

Salgado, A.P.S.P.; Cardoso, M.G.; Souza, P.E.; Souza, J.A.; Abreu, C.M.P.; Pinto, J.E.B.P. 2003. Avaliação da atividade fungitóxica de óleos essenciais de folhas de Eucalyptus sobre Fusarium oxysporum, Botrytis cinerea e Bipolares sorokiniana. Ciência e Agrotecnologia, 27: 249-254.

Simões, C.M.; Shenkel, E.P.; Gosman, G.; Mello, J.C.P.; Mentz, L.A.; Petrovick, P.R. 2004. Farmacognosia: da planta ao medicamento. Porto Alegre: Ed. Da UFRGS; Florianópolis: Ed.5 Da UFSC, Brasil. 1102pp.

Recebido em 27/06/2008

Aceito em 25/09/2008 
